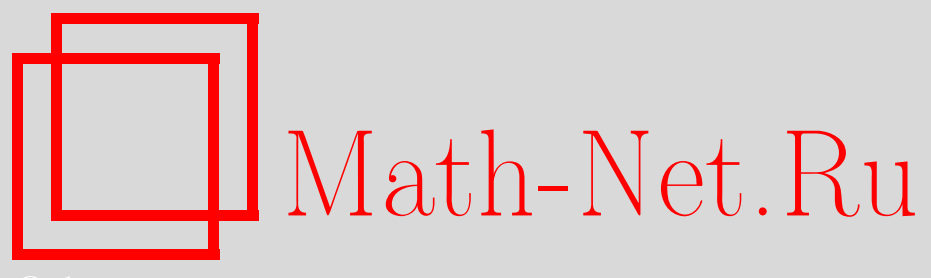

Е. М. Варфоломеев, О бифуркации Андронова-Хопфа для квазилинейных параболических функциональнодифференциальных уравнений с преобразованиями пространственных переменных, УМН, 2007, том 62, выпуск $2,173-174$

DOI: https://doi.org/10.4213/rm6389

Использование Общероссийского математического портала Math-Net.Ru подразумевает, что вы прочитали и согласны с пользовательским соглашением http: //www . mathnet.ru/rus/agreement

Параметры загрузки:

IP : 54.224 .60 .19

26 апреля 2023 г., 13:06:55

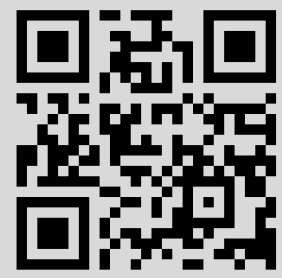




\section{О бифуркации Андронова-Хопфа для квазилинейных параболических функционально-дифференциальных уравнений с преобразованиями пространственных переменных}

\section{Е. М. Варфоломеев}

Нелинейная оптическая система с преобразованиями поля в двумерной обратной связи описывается следующей задачей Неймана [1]:

$$
\begin{gathered}
u_{t}+u=D \Delta u+K\left(1+\sum_{i=1}^{N} \gamma_{i} \cos u_{g_{i}}\right), \quad x \in Q, \quad t \in \mathbb{R}, \\
\left.(\partial u / \partial \widetilde{\nu})\right|_{\partial Q \times \mathbb{R}}=0 .
\end{gathered}
$$

Здесь $Q \subset \mathbb{R}^{n}$ - ограниченная область с границей $\partial Q \in C^{\infty} ; D, K, \gamma_{1}, \ldots, \gamma_{N} \in \mathbb{R}$ постоянные коэффициенты, не равные нулю; $u_{g_{i}}=u\left(g_{i}(x), t\right), g_{i}: \bar{Q} \rightarrow g_{i}(\bar{Q})$ - взаимно однозначные преобразования, $i=1, \ldots, N$; $\nu$ - единичный вектор внешней нормали к $\partial Q$ в точке $x, \widetilde{\nu}=(\nu, 0)$.

$\mathrm{C}$ точки зрения приложений представляет интерес изучение бифуркации Андронова-Хопфа периодических решений указанного уравнения. Во многих работах (см., например, [2], [3]) эта задача рассматривалась при условии, что область $Q$ - окружность или круг с одним преобразованием переменных в виде вращения на постоянный угол и сжатия. Случай произвольных области $Q$ и одного преобразования переменных изучался в работе [4]. В работе [5] использовалась нормальность линеаризованного оператора задачи. Нормальность такого оператора в случае двух преобразований переменных рассматривалась в работе [6].

В настоящей работе обобщены результаты работы [4] на случай конечного числа преобразований переменных. Для этого используются методы исследования бифуркации Андронова-Хопфа в бесконечномерных задачах, развитые в работах [7], [8].

УСловие 1. $g_{i}(Q) \subseteq Q, g_{i}(x) \not \equiv x(x \in Q), i=1, \ldots, N$.

Условие 2. Операторы $G_{i}: L_{p}(Q) \rightarrow L_{p}(Q),\left(G_{i} u\right)(x)=u\left(g_{i}(x)\right), i=1, \ldots, N$, ограничены.

Решение $w$ задачи (1), (2) называется пространственно-однородным стационарным решением, если оно не зависит от $x \in Q$ и $t \in \mathbb{R}$. Оно удовлетворяет трансцендентному уравнению

$$
w=K\left(1+\sum_{i=1}^{N} \gamma_{i} \cos w\right) .
$$

УСловиЕ 3. $1+\widehat{K} \sin \widehat{w} \sum_{i=0}^{N} \gamma_{i} \neq 0$, где $\widehat{w}$ - решение уравнения (3) для $K=\widehat{K}$.

Обозначим через $C_{2 \pi}^{\sigma}(X)$ пространство всех $\sigma$-непрерывных по Гёльдеру $2 \pi$-периодических функций $\varphi: \mathbb{R} \rightarrow X$ с нормой

$$
\|\varphi\|_{C_{2 \pi}^{\sigma}(X)}=\sup _{0 \leqslant t \leqslant 2 \pi}\|\varphi(t)\|_{X}+\sup _{0 \leqslant s<t \leqslant 2 \pi}\|\varphi(t)-\varphi(s)\|_{X} /(t-s)^{\sigma},
$$

где $X$ - вещественное банахово пространство, $0<\sigma<1$.

Пусть $C_{2 \pi}^{1, \sigma}(X)$ - банахово пространство дифференцируемых функций $\varphi: \mathbb{R} \rightarrow X$ таких, что $\varphi$ и $\varphi^{\prime}$ принадлежат $C_{2 \pi}^{\sigma}(X)$ с нормой $\|\varphi\|_{C_{2 \pi}^{1, \sigma}(X)}=\sup _{0 \leqslant t \leqslant 2 \pi}\|\varphi(t)\|_{X}+$ $\left\|\varphi^{\prime}\right\|_{C_{2 \pi}^{\sigma}(X)}$.

Обозначим через $W_{p}^{k}(Q)\left(\widetilde{W}_{p}^{k}(Q)\right)$ пространство Соболева вещественнозначных (комплекснозначных) функций с обобщенными производными вплоть до порядка $k$

Работа выполнена при поддержке РФФИ (грант № 04-01-00256). 
из $L_{p}(Q)\left(\widetilde{L}_{p}(Q)\right) . \quad$ Пусть $W_{p, N}^{2}(Q)=\left\{v \in W_{p}^{2}(Q):\left.(\partial v / \partial \nu)\right|_{\partial Q}=0\right\}, \widetilde{W}_{p, N}^{2}(Q)=$ $\left\{v \in \widetilde{W}_{p}^{2}(Q):\left.(\partial v / \partial \nu)\right|_{\partial Q}=0\right\}$.

Положим $K=\widehat{K}+\varkappa$. Пусть $w=w(\varkappa)$ удовлетворяет уравнению (3) для $K=\widehat{K}+\varkappa$ и $w(0)=\widehat{w}$. Представим решение задачи $(1),(2)$ в виде $u(x, t, \varkappa)=w(\varkappa)+v(x, t, \varkappa)$. Уравнение (1) примет вид

$$
v_{t}=f(v, \varkappa)
$$

где $f(v, \varkappa)=D \Delta v-v+(\widehat{K}+\varkappa) \sum_{i=1}^{N} \gamma_{i}\left(\cos \left(w(\varkappa)+v_{g_{i}}\right)-\cos w(\varkappa)\right)$.

Введем оператор $\mathscr{L}(\varkappa): \mathscr{D}(\mathscr{L}(\varkappa)) \subset L_{p}(Q) \rightarrow L_{p}(Q)$ с областью определения $\mathscr{D}(\mathscr{L}(\varkappa))=W_{p, N}^{2}(Q)$ по формуле $\mathscr{L}(\varkappa) v=f_{v}(0, \varkappa) v=D \Delta v-v-(\widehat{K}+\varkappa) \sin w(\varkappa) \times$ $\sum_{i=1}^{N} \gamma_{i} v_{g_{i}}$. Рассмотрим оператор $\widetilde{\mathscr{L}}(\varkappa): \mathscr{D}(\widetilde{\mathscr{L}}(\varkappa)) \subset \widetilde{L}_{p}(Q) \rightarrow \widetilde{L}_{p}(Q)$ с областью определения $\mathscr{D}(\widetilde{\mathscr{L}}(\varkappa))=\widetilde{W}_{p, N}^{2}(Q)$, заданный по формуле $\widetilde{\mathscr{L}}(\varkappa)=\mathscr{L}(\varkappa) v_{1}+i \mathscr{L}(\varkappa) v_{2}$, где $v_{1}, v_{2} \in \mathscr{D}(\mathscr{L}(\varkappa)), v=v_{1}+i v_{2}$.

Обозначим через $\lambda_{s}(\varkappa)=\delta_{s}(\varkappa)+i \omega_{s}(\varkappa), s=1,2, \ldots$, собственные значения оператора $\widetilde{\mathscr{L}}(\varkappa)$.

Условие 4. $\lambda_{1}(0)=i \widehat{\omega}-$ простое собственное значение оператора $\widetilde{\mathscr{L}}(0): \mathscr{D}(\widetilde{\mathscr{L}}(\varkappa))$ $\subset \widetilde{L}_{p}(Q) \rightarrow \widetilde{L}_{p}(Q)$, причем $\widehat{\omega}>0, k \widehat{\omega} i \notin \sigma(\widetilde{\mathscr{L}}(0)), k=0,2,3, \ldots, u \delta_{1}^{\prime}(0) \neq 0$.

Положим $\tau=\widehat{\omega} \omega(\varkappa) t$, где $\omega(\varkappa)$ - неизвестная частота, близкая к 1 , и рассмотрим $2 \pi$-периодические решения уравнения

$$
v_{\tau}=(\widehat{\omega} \omega(\varkappa))^{-1} f(v(\tau), \varkappa), \quad \tau \in \mathbb{R} .
$$

Основным результатом работы является следующая теорема.

Теорема 1. Пусть выполняются условия 1-4. Зафиксируем $\sigma \in(0,1)$ и $p>n / 2$.

Тогда существуют $\varepsilon_{0}>0$, аналитическая вектор-функиия $\varepsilon \mapsto(v(\varepsilon), \omega(\varepsilon), \varkappa(\varepsilon))$ из $\left(-\varepsilon_{0}, \varepsilon_{0}\right)$ в $C_{2 \pi}^{\sigma}\left(W_{p, N}^{2}(Q)\right) \cap C_{2 \pi}^{1, \sigma}\left(L_{p}(Q)\right) \times \mathbb{R} \times \mathbb{R}$ такал, ито $v(0)=0, \omega(0)=1$, $\varkappa(0)=0$ u $v(\varepsilon)$ не постоянна по $\tau$ при $\varepsilon \neq 0$, и аналитическая функция $\varepsilon \mapsto w(\varkappa(\varepsilon))$ из $\left(-\varepsilon_{0}, \varepsilon_{0}\right)$ в $\mathbb{R}$.

Функиия $u(x, t, \varepsilon)=w(\varkappa(\varepsilon))+v(x, \tau, \varepsilon)$ является $2 \pi(\widehat{\omega} \omega(\varepsilon))^{-1}$-периодическим по $t$ решением задачи (1), (2), где $\tau=\omega(\varepsilon) \widehat{\omega} t$. При этом $\omega(\varepsilon)=1+\varepsilon^{2} \omega_{2}+\varepsilon^{3} \omega_{3}+\cdots$, $\varkappa(\varepsilon)=\varepsilon^{2} \varkappa_{2}+\varepsilon^{3} \varkappa_{3}+\cdots$.

Более того, существует $\delta_{0}>0$ такое, что если $\bar{\varkappa}, \bar{\omega} \in \mathbb{R} u \bar{v} \in C_{2 \pi}^{\sigma}\left(W_{p, N}^{2}(Q)\right) \cap$ $C_{2 \pi}^{1, \sigma}\left(L_{p}(Q)\right)$ удовлетворяют условиям

$$
\begin{gathered}
\bar{v}^{\prime}(\tau)=(\widehat{\omega} \bar{\omega})^{-1} f(\bar{v}(\tau), \bar{\varkappa}), \quad \tau \in \mathbb{R}, \\
\|\bar{v}\|_{C_{2 \pi}^{\sigma}\left(W_{p, N}^{2}(Q)\right) \cap C_{2 \pi}^{1, \sigma}\left(L_{p}(Q)\right)}<\delta_{0}, \quad|\bar{\varkappa}|<\delta_{0}, \quad|1-\bar{\omega}|<\delta_{0},
\end{gathered}
$$

то существуют $\theta \in[0,2 \pi) u \varepsilon \in\left(-\varepsilon_{0}, \varepsilon_{0}\right)$ такие, что $\bar{\varkappa}=\varkappa(\varepsilon), \bar{\omega}=\omega(\varepsilon), \bar{v}(\tau)=$ $v(x, \tau+\theta, \varepsilon)$.

Автор глубоко благодарен проф. А.Л. Скубачевскому за постановку задачи и постоянное внимание к работе.

\section{Список литературы}

[1] М. А. Воронцов, Ю. Д. Думаревский, Д. В. Пруидзе, В. И. Шмальгаузен, Изв. АН СССР. Сер. физ., 52:2 (1988), 374-376. [2] Е. П. Белан, О. Б. Лыкова, Дифферени. уравнения, 40:10 (2004), 1348-1357. [3] А. В. Разгулин, ЖКВМ и МФ, 33:1 (1993), 69-80. [4] А. Л. Скубачевский, Дифферени. уравнения, 34:10 (1998), 1394-1401. [5] A.L. Skubachevskii, Nonlinear Anal., 32:2 (1998), 261-278. [6] Е. М. Варфоломеев, УМH, 61:1 (2006), 173-174. [7] M. G. Crandall, P. H. Rabinowitz, Arch. Rational Mech. Anal., 67:1 (1977), 53-72. [8] G. Da Prato, A. Lunardi, Ann. Inst. H. Poincaré Anal. Non Linéaire, 3:4 (1986), 315-329.

Е. М. Варфоломеев (Е. M. Varfolomeev)

Российский университет дружбы народов

E-mail: varfolomeyev@mail.ru
Представлено В.М.Бухштабером Принято редколлегией 27.12 .2006 